\title{
Effect of Various Process Parameters on Morphology, Rheology, and Polygalacturonase Production by Aspergillus sojae in a Batch Bioreactor
}

\author{
Selale Oncu, Canan Tari,* and Sevcan Unluturk \\ Department of Food Engineering, Izmir Institute of Technology, Urla, Izmir 35430, Turkey
}

\begin{abstract}
The effects of $\mathrm{pH}$, agitation speed, and dissolved oxygen tension (DOT), significant in common fungal fermentations, on the production of polygalacturonase (PG) enzyme and their relation to morphology and broth rheology were investigated using Aspergillus sojae in a batch bioreactor. All three factors were effective on the response parameters under study. An uncontrolled $\mathrm{pH}$ increased biomass and PG activity by $27 \%$ and $38 \%$, respectively, compared to controlled $\mathrm{pH}$ (pH 6) with an average pellet size of $1.69 \pm 0.48 \mathrm{~mm}$. pH did not significantly affect the broth rheology but created an impact on the pellet morphology. Similarly, at constant agitation speed the maximum biomass obtained at $500 \mathrm{rpm}$ and at $30 \mathrm{~h}$ was 3.27 and 3.67 times more than at 200 and $350 \mathrm{rpm}$, respectively, with an average pellet size of $1.08 \pm 0.42 \mathrm{~mm}$. The maximum enzyme productivity of $0.149 \mathrm{U} \mathrm{mL}^{-1} \mathrm{~h}^{-1}$ was obtained at $200 \mathrm{rpm}$ with an average pellet size of $0.71 \pm 0.35 \mathrm{~mm}$. Non-Newtonian and pseudoplastic broth rheology was observed at $500 \mathrm{rpm}$ agitation speed, broth rheology exhibited dilatant behavior at the lower agitation rate $(200 \mathrm{rpm})$, and at the medium agitation speed $(350 \mathrm{rpm})$ the broth was close to Newtonian. Furthermore, a DOT range of 30-50\% was essential for maximum biomass formation, whereas only $10 \%$ DOT was required for maximum PG synthesis. Non-Newtonian shear thickening behavior $(n>1.0)$ was depicted at DOT levels of $10 \%$ and $30 \%$, whereas non-Newtonian shear thinning behavior $(n<1.0)$ was dominant at 50\% DOT. The overall fermentation duration $(50-70 \mathrm{~h})$ was considerably shorter compared to common fungal fermentations, revealing the economic feasibility of this particular process. As a result this study not only introduced a new strain with a potential of producing a highly commercially significant enzyme but also provided certain parameters significant in the design and mathematical modeling of fungal bioprocesses.
\end{abstract}

\section{Introduction}

Deep tank fermentations are widely used in the production of enzymes, antibiotics, and organic acids, which find many applications in the food, medicine, pharmaceutical, chemical, and textile industries (1). The diverse range of commercially exploited fungal products, which have a large contribution to the global economy, is expanding enormously. However, their filamentous growth characteristic creates a number of process engineering problems attributed to the morphological change encountered during the fermentation process at large scale (2). This affects the maximum product concentration and the productivity of the organism. It is well documented that the fungal culture exhibits two major morphologies observed as pellets, which are spherical agglomerates of hyphaes, or mycelia, which is free mycelium dispersed throughout the culture medium (3). These morphologies are very much determined by several environmental and genetic factors including type of organism, $\mathrm{pH}$ and composition of the media, inoculation ratio and type of the inoculum, agitation speed and aeration rate, feeding rate, and genetic factors of the culture (3-6). In industrial fermentations fungal morphology is often considered as one of the key parameters of physiological and engineering studies of filamentous fermentations used in the design and operation of such systems (6). Complex interactions of various operating conditions such as agitation intensity, aeration rate, type of microbial

* To whom correspondence should be addressed. Email: canantari@iyte.edu.tr. species, media composition and supply, and the hydrodynamic regime in the bioreactor determine the metabolic performance of a microbial culture, which in turn determines the cellular morphology affecting the bulk broth rheology. Rheology in turn affects the supply of nutrients and oxygen, and the ease of mixing of the broth. Rheology-morphology relationships are particularly significant in fermentations involving filamentous fungi $(7,8)$. However, excessive hydrodynamic shear stresses may damage mycelial hyphae and pellets, where much lower shear stresses are sufficient to influence growth morphology. A pellet type of morphology is usually preferred in industrial fermentations and in downstream processing because of the nonviscous rheology of the broth $(9,10)$. In such fermentations, the mass transfer of oxygen and nutrients is considerably better and the subsequent separation of the pellets from the medium is simpler (4). Since agitation and aeration are also much easier, the power input and therefore the operating cost are lower. However, in fermentations where the mycelia form is dominant and the cell growth and productivity are higher, the broth is much more viscous, resulting in heterogeneous stagnant nonmixed zone formations, which are harder and more expensive to operate (7). Hence, the morphology of the culture, which affects productivity and results in rheological changes of the broth, needs to be controlled. Therefore, the relationship between morphology and rheology and the factors influencing them have to be fully investigated. Such knowledge can be very valuable in the optimization and design of common industrial fungal fermentations. 
With this perspective, polygalacturonase, which attracts the most attention among the pectinases due to its wide application area, is considered as a model product produced by a new organism Aspergillus sojae, which was not considered for this purpose so far to unveil the common industrial problem facing fungal fermentations. Another objective of the study was to extend the previous shake flask studies, which determined the high polygalacturonase potential of this organism to bioreactor studies $(11-13)$. It is well known that as opposed to shake flask studies, bioreactors are a better controlled environment where the effect of common factors such as $\mathrm{pH}$, agitation, and dissolved oxygen tension can be observed and controlled. They also provide information required in scaleup processes. To the best of our knowledge there is no available literature on the polygalacturonase production of this organism in batch bioreactors; therefore this study will be one of initial studies using this organism in the production of a highly valuable product and a new reference point in the microbiology area and enzyme industry. Also there is no literature that we know about on the investigation of the relationship of morphology and rheology to polygalacturonase production using other organisms. Hence, it will close to some degree the gap in the literature, which has been lacking so far.

As is known because of their myriad applications, the biotechnological potential of pectolytic enzymes from microorganisms has drawn a great deal of attention worldwide (14). These are used mainly in the food industry in the clarification of fruit and vegetable juice, maceration of vegetables and fruits, extraction of olive oil and in the treatment of raw fibers in the textile and paper industry and in the process of waste treatment (15). It is also reported that pectinases, as one of the upcoming enzymes in the commercial sector, contribute to almost $25 \%$ of global enzyme sales, and its contribution is estimated to increase further by the year 2009 (16). In order to meet this high demand, it is of great importance to produce this enzyme in a cost-effective and productive way, which makes this study extra novel.

Therefore, combining the two main objectives in a single study, the effect of process parameters significant to fungal fermentations such as $\mathrm{pH}$, agitation speed, and dissolved oxygen concentration on pellet morphology, broth rheology, and polygalacturonase production were investigated in the submerged fermentation of $A$. sojae.

\section{Materials and Methods}

Microorganism. Aspergillus sojae ATCC 20235 was purchased from Promochem Inc., an international distributor of ATCC (American Type Culture Collection) in Europe. The propagation of this culture was done on YME agar slant medium containing malt extract at $10 \mathrm{~g} / \mathrm{L}$, yeast extract at $4 \mathrm{~g} / \mathrm{L}$, glucose at $4 \mathrm{~g} / \mathrm{L}$, and agar at $20 \mathrm{~g} / \mathrm{L}$ concentrations incubated at $30{ }^{\circ} \mathrm{C}$ until well sporulated (1 week). Stock cultures of this strain were prepared with $20 \%$ glycerol water and stored at $-80{ }^{\circ} \mathrm{C}$.

Inoculum Preparation. For the seed medium, molasses medium formulation composed of glycerol (45 g/L), peptone (18 g/L), molasses (45 g/L), $\mathrm{NaCl}(5 \mathrm{~g} / \mathrm{L}), \mathrm{FeSO}_{4} \cdot 7 \mathrm{H}_{2} \mathrm{O}$ $(15 \mathrm{mg} / \mathrm{L}), \mathrm{KH}_{2} \mathrm{PO}_{4}(60 \mathrm{mg} / \mathrm{L}), \mathrm{MgSO}_{4}(50 \mathrm{mg} / \mathrm{L}), \mathrm{CuSO}_{4} \cdot$ $5 \mathrm{H}_{2} \mathrm{O}(12 \mathrm{mg} / \mathrm{L}), \mathrm{MnSO}_{4} \cdot \mathrm{H}_{2} \mathrm{O}(15 \mathrm{mg} / \mathrm{L})$, and agar $(20 \mathrm{~g} / \mathrm{L})$ was used. Initially, the frozen stock cultures were inoculated on YME agar and incubated for 1 week at $30{ }^{\circ} \mathrm{C}$ for activation. After this period a single isolate of the strain was inoculated on a molasses slant and incubated for another week at the same temperature. Following the incubation period, $5 \mathrm{~mL}$ of $0.01 \%$ $(\mathrm{v} / \mathrm{v})$ tween 80 solution was added into the slant, harvested into an empty sterile falcon tube, and tested for sterility, viability, and spore count. The spore counts were performed using a Thoma bright line hemacytometer (Marienfield, Germany). The suspensions were stored at $4{ }^{\circ} \mathrm{C}$ and used as inoculum for the fermentation process.

Production Medium and Fermentation. The fermentation was carried out in a $5 \mathrm{~L}$ bioreactor (New Brunswick BioFlo $3000, \mathrm{NJ}$ ) with a working volume of $4 \mathrm{~L}$ at $30^{\circ} \mathrm{C}$ and $2.5 \mathrm{~L} / \mathrm{min}$ aeration rate for $96 \mathrm{~h}$. Agitation was provided with two sets of standard six-blade rushton impellers $(6 \mathrm{~cm}$ diameter $)$ and four baffle plates, equipped in the bioreactor (the distance between the two impellers was $108 \mathrm{~mm}$ and the distance to bottom impeller was $39 \mathrm{~mm})$. The fermentation medium (4 L) was composed of corn steep liquor $(5 \mathrm{~g} / \mathrm{L})$, peptone $(5 \mathrm{~g} / \mathrm{L})$, maltrin 50 (75 g/L), disodium phosphate (3.2 g/L), monosodium phosphate $(3.3 \mathrm{~g} / \mathrm{L})$, and glucose $(0.75 \mathrm{~g} / \mathrm{L})$. The bioreactor was inoculated with a total of $2 \times 10^{6}$ spores as prepared in the inoculum preparation section. The $\mathrm{pH}$ of the fermenter medium was adjusted automatically to $\mathrm{pH} 6$ using $6 \mathrm{~N} \mathrm{NaOH}$ or $10 \%$ $\mathrm{H}_{2} \mathrm{SO}_{4}$ at the onset of the fermentation and was not controlled otherwise stated. Dissolved oxygen tension was monitored by means of a polarographic oxygen electrode (Mettler Toledo, USA) on line. Prior to each fermentation, the bioreactor was saturated to $100 \%$ with air. Samples were taken periodically and assayed for enzyme activity, biomass determination, and substrate utilization. Enzyme activity and carbohydrate content were determined on supernatant obtained by centrifugation of the broth at $5000 \mathrm{rpm}$ for $15 \mathrm{~min}$. Fungal morphology and broth rheology were investigated at the end of the fermentation period (at $96 \mathrm{~h}$ ).

Enzyme Assay. PG (polygalacturonase) activity was assayed according to the procedure given by Panda et al. (17) by using $2.4 \mathrm{~g} / \mathrm{L}$ of polygalacturonic acid as substrate at $\mathrm{pH} 6.6$ and $26^{\circ} \mathrm{C}$. The amount of substrate and enzymes used were 0.4 and $0.086 \mathrm{~mL}$, respectively. In this study, one unit of enzyme activity was defined as the amount of enzyme that catalyzes the release of $1 \mu \mathrm{mol}$ of galacturonic acid per unit volume of culture filtrate per unit time at standard assay conditions. Galacturonic acid (Sigma, St. Louis, MO) was used as standard for calibration curve of PGase activity.

activity $(\mathrm{U} / \mathrm{mL})=$

$(\mathrm{mg}$ of galacturonic acid/212.12) $\times(1 / 20) \times(1 / 0.086)$

Biomass Determination. The biomass expressed as dry cell weight $(\mathrm{g} / \mathrm{L})$ was determined by means of gravimetric method. The fermentation broth $(25 \mathrm{~mL})$, which was taken periodically during fermentation, was filtered through a preweighed Whatman No.1 filter paper, followed by drying to constant weight at $37{ }^{\circ} \mathrm{C}$ for approximately $24 \mathrm{~h}$.

Protein and Carbohydrate Assay. The total protein contents of the samples were determined according to the method described by Lowry et al. (18). A protein standard calibration curve was prepared in the range of $0-300 \mu \mathrm{g} / \mathrm{mL}$ with the protein standard Bovine Serum Albumin.

The total carbohydrate contents of the samples were determined by the phenol/sulfuric acid method. A carbohydrate standard calibration curve was prepared in the range of 10$40 \mu \mathrm{g} / \mathrm{mL}$ with D-glucose as the standard. The absorbance was read at $490 \mathrm{~nm}$ using a Varian Cary Bio $100 \mathrm{UV}-$ visible spectrophotometer (19).

Morphological and Rheological Measurements. In order to investigate the pellet morphology, $1 \mathrm{~g}$ of fermentation broth was transferred to a petri dish and fixed with an equal volume of fixative $(13 \mathrm{~mL}$ of $40 \%$ formaldehyde, $5 \mathrm{~mL}$ of glacial acetic 
acid, $200 \mathrm{~mL}$ of $50 \%$ ethanol) (20). Pellet morphology was characterized by using image analysis (21). Pellet particles were analyzed for determination of the number of pellet per given volume and pellet size. The image was captured with a eurocam (Euromax, Holland) mounted on a phase contrast microscope (Novex, Holland). Image analysis was performed with the software package Image-Pro Plus 4.5.1. (Media Cybernetics Inc., Silver Spring, MD). The size of the pellet was quantified using the diameter corresponding to a circular area equivalent to the pellet projected area (22).

Rheological properties of the fermentation broth were determined by using a rotational viscometer (Brookfield DV II + Pro, Brookfield Engineering Lab. Inc., MA) equipped with a Rheocalc32 program. The principle of operation of the DV II + Pro was to drive a spindle (which was immersed in the test fluid) through a calibrated spring. The viscous drag of the fluid against the spindle was measured by the spring deflection. Spring deflection was measured with a rotary transducer for a given spindle geometry and speed (23). An increase in viscosity was detected by an increase in deflection of the spring, which allowed measurement of a variety of viscosity ranges. Temperature was adjusted to $30{ }^{\circ} \mathrm{C}$ for all measurements.

Broth rheology of the samples collected in $600 \mathrm{~mL}$ beakers was usually examined at the end of the fermentation period (96 h). For periodic measurements during fermentation, a small amount of broth $(16 \mathrm{~mL})$ was driven from the bioreactor and rheological measurement was performed by using a UL adapter. In samples measured by the UL adapter, the size of pellets was generally of the same order of magnitude as the annulus of the UL adapter. In order to overcome this measurement problem, the measured viscosity of the fermentation broth was assumed to be a suspension and was corrected by using a mathematical model developed by Metzner (24).

The model for estimating viscosity of dilute suspensions was based on volume fraction of the suspended solids (pellet) $(\phi)$ and the relative viscosity of the suspension, given in eq 2 and 3 , where $\eta$ is the viscosity of the suspension and $\eta_{\mathrm{s}}$ is the viscosity of the continuous phase:

$$
\begin{gathered}
\eta_{\mathrm{r}}=\frac{\eta}{\eta_{\mathrm{s}}} \\
\eta_{\mathrm{r}}=\left[1-\left(\frac{\phi}{A}\right)\right]^{-2}
\end{gathered}
$$

In eq $3, A$ was in the range of 0.44 and 0.68 for the crystal and spherical shape particles. For this purpose, the volume and mass of each sample containing spherical pellets was initially measured and the bulk density of the suspension (fermentation broth) was calculated. Then the sample was filtered and mass and volume measurements were repeated for the filtrate (clear broth). Volume fractions $(\phi)(\mathrm{v} / \mathrm{v})$ were calculated from these measurements. After, the viscosity of the clear broth, $\eta_{\mathrm{s}}$, was measured by using a viscometer and the suspension viscosity $\eta$ was calculated from eq 2 .

All of the shear data were then analyzed according to the power law (eq 4) and the Herschel-Bulkley model (eq 5) (25):

$$
\begin{gathered}
\tau=K \gamma^{n} \\
\tau=\tau_{0}+K \gamma^{n}
\end{gathered}
$$

where $\tau_{0}, \tau$, and $\gamma$ are the yield stress, shear stress, and shear rate, respectively. The constants $K$ and $n$ represent the consistency index $\left(\mathrm{Pa} \cdot \mathrm{s}^{n}\right)$ and the flow behavior index. Taking the (a)
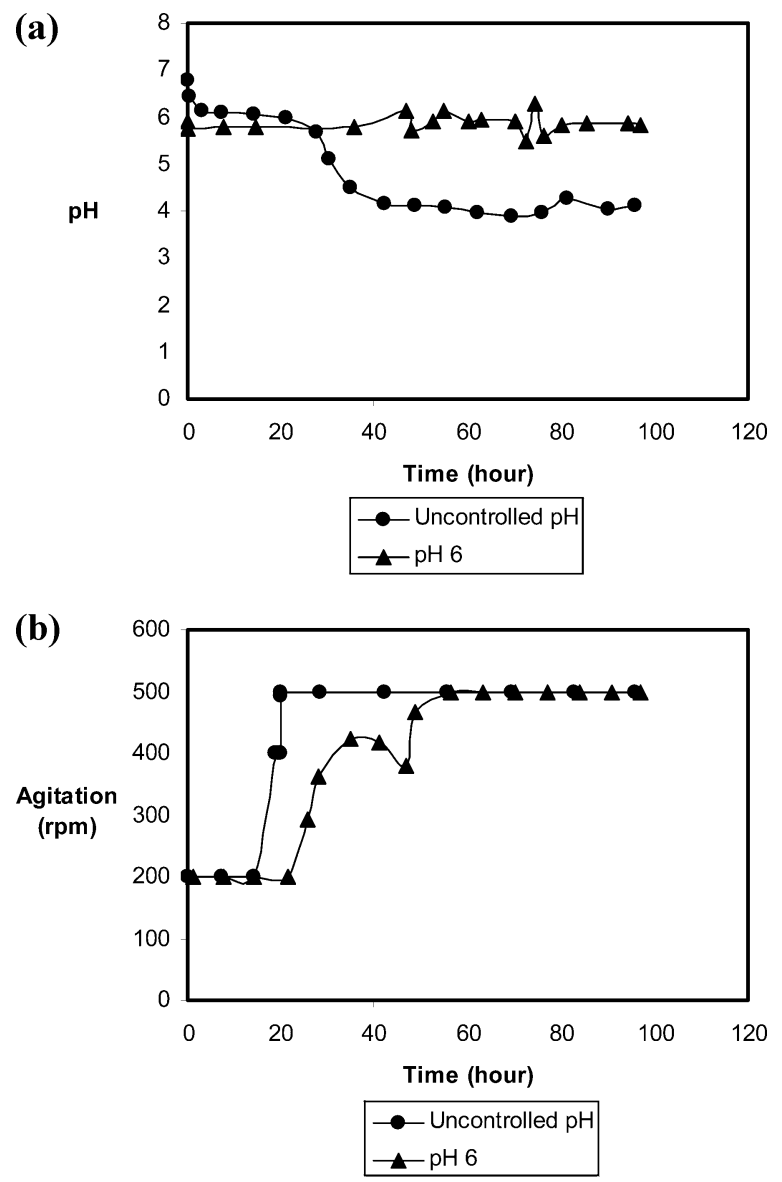

Figure 1. Profiles of (a) $\mathrm{pH}$ and (b) agitation of uncontrolled and constant $\mathrm{pH} 6$ experiments conducted at $30^{\circ} \mathrm{C}, 2.5 \mathrm{~L} / \mathrm{min}$ air flow rate and cascaded between 200 and $500 \mathrm{rpm}$ agitation speed.

logarithm on both sides of eqs 4 and 5 yields $K$ and $n$ values. Since extra force $\left(\tau_{0}\right)$ was needed to start the flow in the fermentations where the number of pellets were very high, eq 5 was preferred to explain the rheological behavior of the broth.

\section{Results and Discussion}

Effect of Medium pH on Biomass, Polygalacturonase, Pellet Morphology, and Broth Rheology. In order to investigate the effect of $\mathrm{pH}$ as a key parameter significant in fungal fermentations, two types of fermentations were run at constant $\mathrm{pH}$ of 6 and under uncontrolled conditions (initial $\mathrm{pH}$ was adjusted to 6 but afterward not controlled). The fermentation conditions were such that dissolved oxygen tension (DOT) was controlled at $50 \%$ saturation by cascading the agitation speed between the ranges of $200-500 \mathrm{rpm}$ at constant airflow of $2.5 \mathrm{~L} / \mathrm{min}$. The corresponding profiles of $\mathrm{pH}$ and agitation speed are presented in Figure $1(\mathrm{a}, \mathrm{b})$, whereas the profiles of biomass,carbohydrate utilization and pellet size distribution are presented in Figure $2(\mathrm{a}-\mathrm{c})$. The uncontrolled $\mathrm{pH}$ profile (Figure 1a) dropped to $\mathrm{pH} 4$ at the end of $96 \mathrm{~h}$. Comparing this profile with biomass formation and carbohydrate utilization profiles (Figure 2, a and b), the following conclusions can be withdrawn: (1) Uncontrolled $\mathrm{pH}$ resulted in better and faster carbohydrate utilization as a result of better solubility of the nutrient compounds in the broth due to $\mathrm{pH}$ effect. (2) Cell membrane permeability was affected because of low $\mathrm{pH}$ and nutrient transport into the cells was more efficient. In fact maximum biomass that was 4 times higher compared to that of the controlled $\mathrm{pH}$ experiment was achieved at $40 \mathrm{~h}$. The reason for the low biomass (at constant $\mathrm{pH} \mathrm{6)}$ was explained with the 

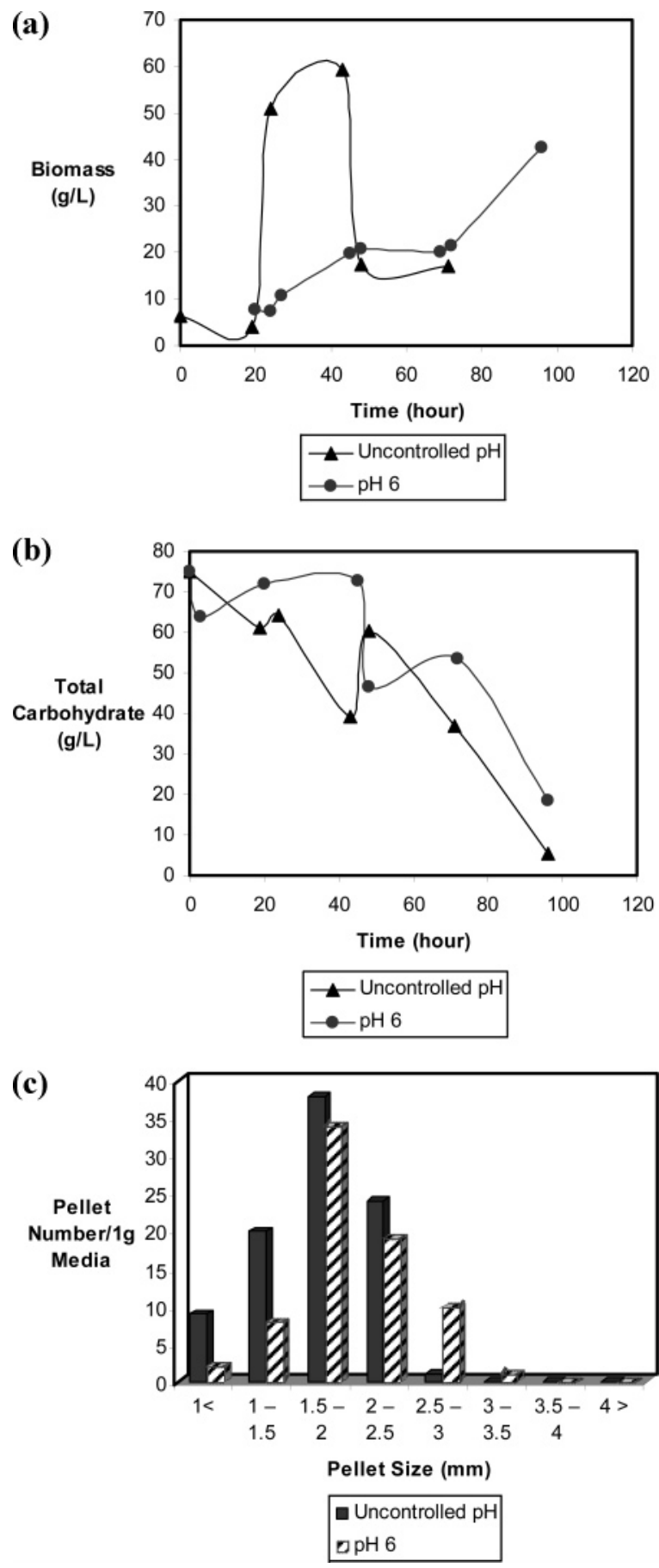

Figure 2. Profiles of (a) biomass, (b) carbohydrate utilization, and (c) pellet size distribution of uncontrolled and constant $\mathrm{pH} 6$ experiments conducted at $30^{\circ} \mathrm{C}, 2.5 \mathrm{~L} / \mathrm{min}$ air flow rate and cascaded between 200 and $500 \mathrm{rpm}$ agitation speed.

small compact and very dense smooth pellet structure (Table 1) possibly causing nutrient and oxygen limitations to the interior of the pellets and reducing the growth. The overall biomass profile was such that controlled $\mathrm{pH}(\mathrm{pH} 6)$ resulted in a gradual increase, whereas uncontrolled $\mathrm{pH}$ resulted in biomass decrease after $45 \mathrm{~h}$. This could be attributed partly to the $\mathrm{pH}$ changes encountered during this period but also to the high agitation speed observed at $500 \mathrm{rpm}$ in order to maintain the dissolved oxygen tension at $50 \%$ (Figure 1b) that exhibited shear effect and possibly mechanical damage to the cells. Hence, the decrease in DOT was faster in the uncontrolled $\mathrm{pH}$ experiments as a result of faster growth increasing the oxygen consumption rate, leading to an earlier start of the cascade. The combination of all of these factors could have contributed to the smaller size and higher number of pellet formation (Table 1 and Figure 2c) observed in the case of uncontrolled $\mathrm{pH}$ experiments. Hence, this observation pointed out some critical information on the mechanism of pellet formation, which was due to chipping off and fragmentation of the existing pellets. However, this cannot be attributed solely in case of controlled $\mathrm{pH}$ experiments where larger and fewer pellets were formed.

The effect of $\mathrm{pH}$ on morphology and its relation to rheology was also investigated (Table 1). Pellet volume fraction and number of pellet formation were comparable; the variation among the suspension viscosities was not extremely significant in both cases in spite of slightly higher $(4.2 \mathrm{cP})$ suspension viscosity when $\mathrm{pH}$ was set to 6 . This was due to the filtrate viscosity, which was found to be faintly higher, imposing an effect on the suspension viscosity calculations. The difference between the filtrate viscosities could also be attributed to the production and secretion of some unknown metabolites into the medium by this microorganism. Overall, $\mathrm{pH}$ did not significantly affect the suspension viscosity, average pellet size, pellet number, and pellet size distribution (Figure 2c) but created an impact on the pellet morphology. Morphology varied between the pellet form with a hairy region surrounding a dense core to a compact, smooth, and very dense pellet structure.

The polygalacuronase profiles (Figure 3 ) for the two experiments were similar in profile but differed in quantities. The maximum polygalacturonase activity was achieved around $45 \mathrm{~h}$ for both cases. The uncontrolled $\mathrm{pH}$ experiments resulted in $38 \%$ more activity compared to that of the controlled one. This could be related to the higher biomass and higher number of pellets obtained. However, in both experiments the activity declined after this period and no activity could be detected at the end of the fermentation ( $96 \mathrm{~h}$ ). Explanations for this can be that either this organism produced proteases that hydrolyzed the enzyme or the medium $\mathrm{pH}$ was not suitable to maintain the stability of the enzyme. Since the proteolytic activity was not determined, the former statement could not be justified, but it is known to be very common among these microorganisms. Aspergillus sojae is considered as GRAS (generally considered as safe) and very popular in Koji fermentation of a Japanese food in solid-state fermentation of soya sources $(26,27)$. Therefore, it is very likely that it might produce high amounts of proteases. However this issue will be exploited in future experiments. As a conclusion, it is recommended to run the fermentation under uncontrolled $\mathrm{pH}$ conditions and stop the run around $50 \mathrm{~h}$ in order to recover the polygalacturonase enzyme. This is actually a positive outcome since not only will the operational costs be reduced as a result of a shorter run but also the acid and base consumption will be eliminated, therefore reducing the overall material cost. On this basis, the forthcoming experiments were run under uncontrolled $\mathrm{pH}$ conditions.

Since there is no literature either on this organism or on the submerged polygalacturonase, no direct comparisons could be made. However there are several literatures sources investigating the effect of $\mathrm{pH}$ on morphology and on the fermentation of various products produced by various fungal organisms revealing that the effect of $\mathrm{pH}$ as a key parameter needs to be investigated for each and every single strain under study $(28-31)$.

Effect of Agitation Speed on Biomass, Polygalacturonase, Pellet Morphology, and Broth Rheology. It is reported by a number of researchers that agitation creates shear forces, which affect microorganisms in several ways, causing morphological changes, variation in their growth and product formation, and damage even to the cell structure $(32-34)$. Based on this, 
Table 1. Effect of pH on Rheology and Morphology at $30^{\circ} \mathrm{C}, 2.5 \mathrm{~L} / \mathrm{min}$ Air Flow Rate and Cascaded at 200-500 rpm Agitation Speed

\begin{tabular}{lll}
\hline & \multicolumn{1}{c}{ uncontrolled $\mathrm{pH}$} & $\mathrm{pH}=6.0$ \\
\hline viscosity (cP) (at 73.38 1/s) & 3.20 & 4.20 \\
$\quad$ suspension & 1.04 & 1.79 \\
$\quad$ filtrate & 0.26 & 0.20 \\
pellet volume fraction & $92 \quad$ small pellets with dense core with a fluffy region & small and compact smooth very dense pellets \\
pellets/1 g media & surrounding the core & \\
pellet morphology & $1.69 \pm 0.48$ & $1.95 \pm 0.46$ \\
average pellet size $(\mathrm{mm})$ &
\end{tabular}

fermentation runs at three different but constant agitation speeds of 200 (tip speed $0.628 \mathrm{~m} / \mathrm{s}$ ), 350 (tip speed $1.099 \mathrm{~m} / \mathrm{s}$ ), and $500 \mathrm{rpm}$ (tip speed $1.57 \mathrm{~m} / \mathrm{s}$ ) and constant air flow $(2.5 \mathrm{~L} / \mathrm{min})$, under uncontrolled $\mathrm{pH}$ conditions were performed. All the other fermentation conditions were the same as described in Materials and Methods. The corresponding profiles of DOT, $\mathrm{pH}$, and biomass are presented in Figure $4(\mathrm{a}-\mathrm{c})$ from which it can be seen that the growth was considerably faster at $500 \mathrm{rpm}$ compared to the other agitation speeds. The maximum biomass obtained at this speed was 3.27 and 3.67 times more than 200 and $350 \mathrm{rpm}$ (at $30 \mathrm{~h}$ ), respectively. For all three runs the differences in $\mathrm{pH}$ profiles were encountered mainly during the lag and growth phases, reaching a final $\mathrm{pH}$ of $4-4.5$ at the stationary and death phases. The slow growth at $200 \mathrm{rpm}$ could be related to insufficient oxygen supply, which dropped to $0 \%$ saturation at $16 \mathrm{~h}$, increased to $10 \%$ after $34 \mathrm{~h}$, and remained at this level until the cessation of the run. This finding was also supported by the carbohydrate utilization profile, which was slower at this agitation speed (Figure 5a). At both 200 and 350 rpm carbohydrate was not utilized completely and remained at high levels. Because of faster biomass formation carbohydrate consumption was almost complete at $500 \mathrm{rpm}$. The DOT profile at this speed was very well correlated with both carbohyrate and biomass profiles (Figure $4 \mathrm{a}$ and $\mathrm{c}$ and Figure 5a). The oxygen supply could have been a limiting factor (DOT was $0 \%$ ) at $500 \mathrm{rpm}$ since oxygen transfer rate was almost equal to oxygen consumption rate. Although DOT level was not the limiting factor at $350 \mathrm{rpm}$ (since it was above $50 \%$ until the end of fermentation), some other factors might have contributed to the slow growth discussed above. Hence, this brings up the discussions on the effect of agitation speed on the morphology of the culture and consequently on the broth rheology.

As can be seen from the pellet size distribution (Figure 5b) very small compact spherical pellets with an average size of $0.71 \pm 0.35 \mathrm{~mm}$ were obtained at $200 \mathrm{rpm}$ with narrow size distibution. However, at $500 \mathrm{rpm}$ small fluffy pellets with an

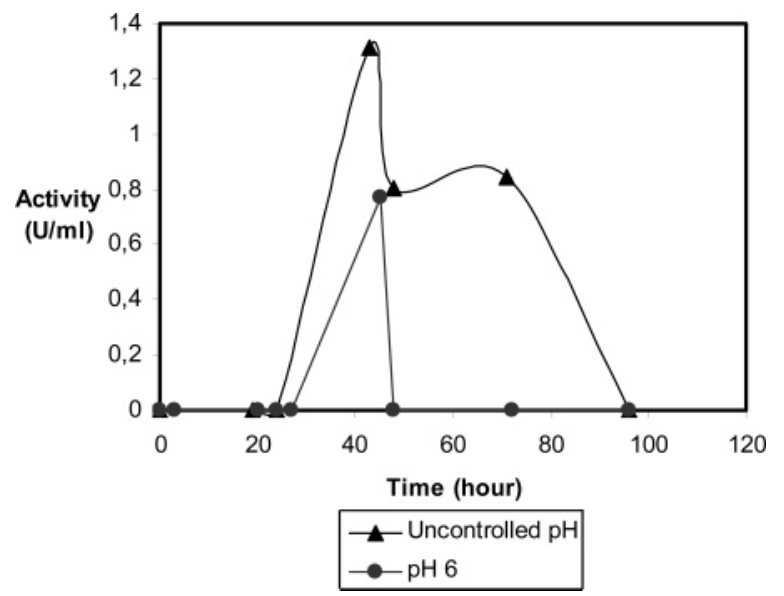

Figure 3. Polygalacturonase profiles of uncontrolled and constant $\mathrm{pH}$ 6 experiments conducted at $30^{\circ} \mathrm{C}, 2.5 \mathrm{~L} / \mathrm{min}$ air flow rate and cascaded between 200 and $500 \mathrm{rpm}$ agitation speed. average size of $1.08 \pm 0.42 \mathrm{~mm}$ with wide pellet size distribution were obtained. Also the mechanism for the pellet formation at this speed as discussed in the previous section was due to chipping off, as can be observed from the pellet size profile taken during the entire run (Figure 5c). As can be observed from this profile, the pellets were formed within $24 \mathrm{~h}$ and the perimeter and core diameter were reduced over the run, pointing out that in addition to the chipping off, the breakage

(a)
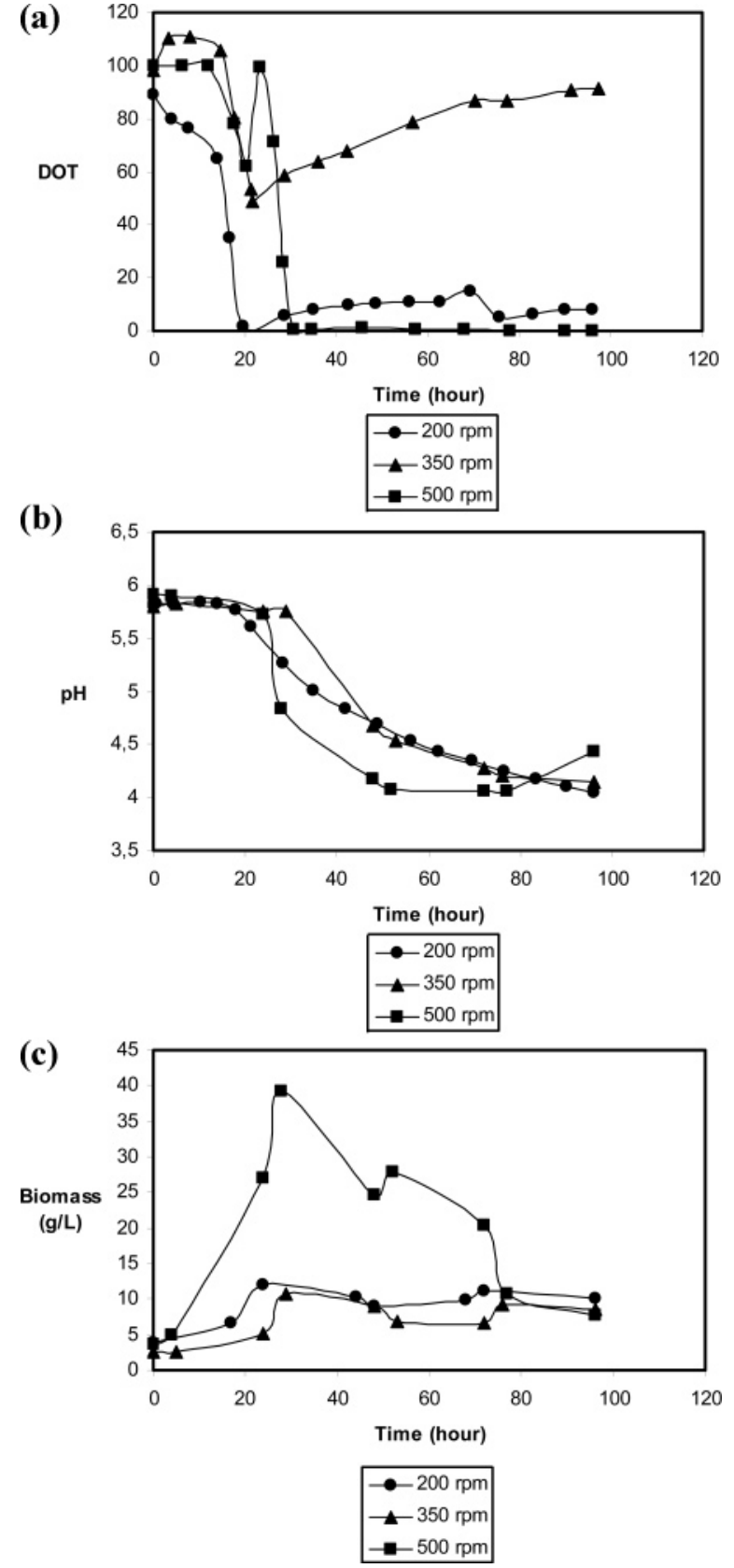

Figure 4. Profiles of (a) DOT, (b) $\mathrm{pH}$, and (c) biomass of experiments conducted at different agitation speed of 200,350 , and $500 \mathrm{rpm}$ at $30{ }^{\circ} \mathrm{C}, 2.5 \mathrm{~L} / \mathrm{min}$ air flow rate. 


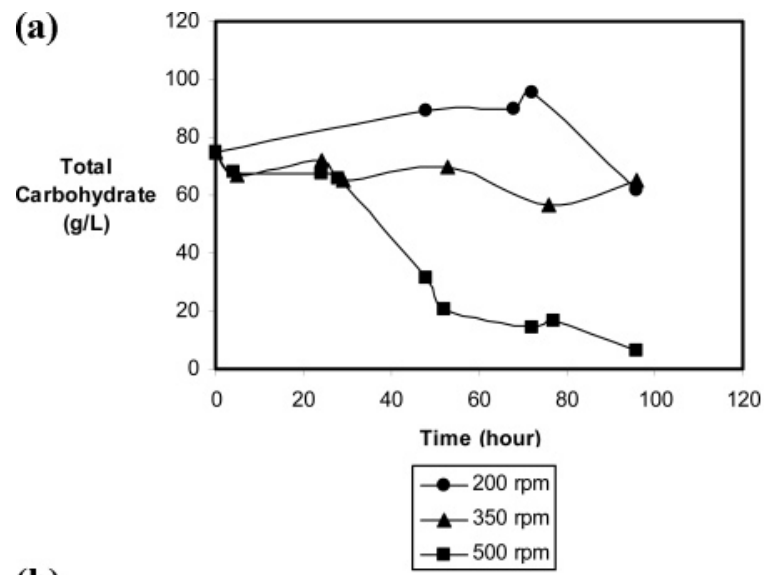

(b)
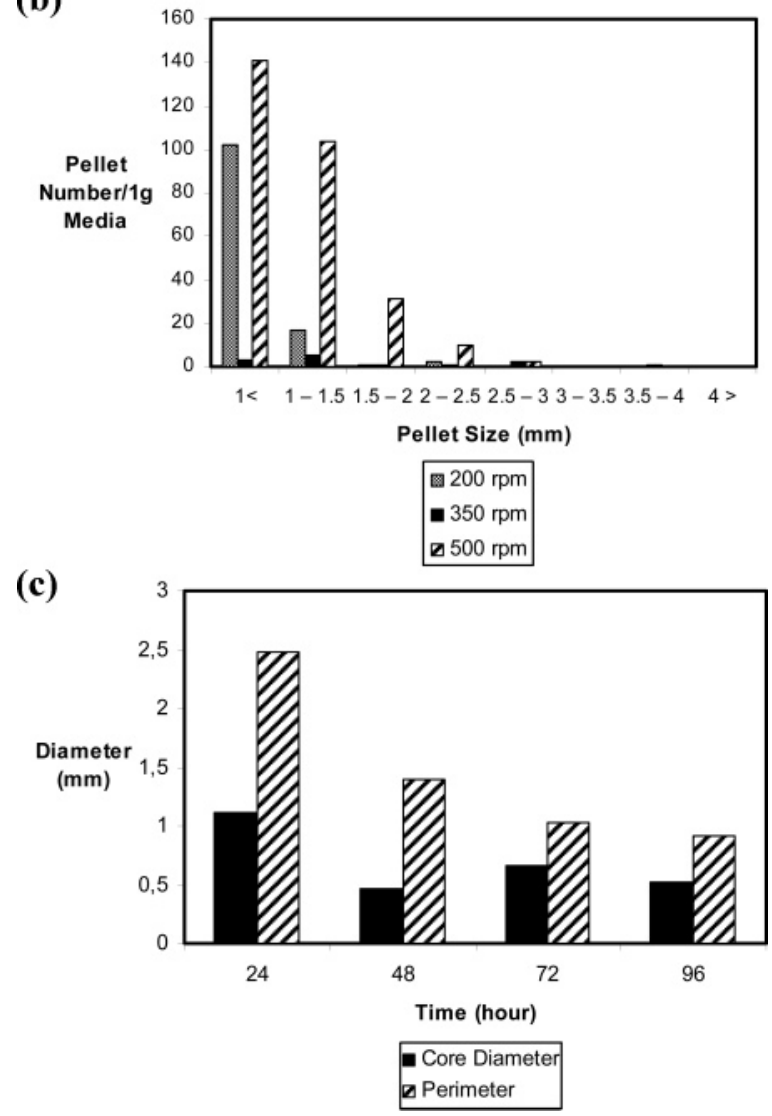

Figure 5. Profiles of (a) carbohydrate utilization and (b) pellet size distribution of experiments conducted at different agitation speed of 200,350 and $500 \mathrm{rpm}$ at $30{ }^{\circ} \mathrm{C}, 2.5 \mathrm{~L} / \mathrm{min}$ air flow rate and (c) pellet size profiles during the course of fermentation conducted at $500 \mathrm{rpm}$.

of pellets might have occurred as well. This explains also the wider size distribution observed in this particular run.

The current observation was in accordance with many reports in the literature, reporting two main mechanisms of pellet size changes: (1) chipping off of pellicles from the surface of pellets and reducing the diameter and (2) direct breakup of the pellet structure due to higher hydrodynamic loads $(2,35)$. Considering the morphology at $350 \mathrm{rpm}$, few big pellets with compact centers with an average size of $1.70 \pm 0.93 \mathrm{~mm}$ were obtained. In fact, this explains that there might have been diffusion mass transfer resistance in the interior of the pellets with respect to oxygen and nutrients. Hence, the DOT level and carbohydrate levels were high, whereas the biomass formation was low (Figure 4a, Figure 5a, and Figure 4c). Furthermore, the mechanism of pellet formation in this case was mostly due to agglomeration of small pellets into bigger ones induced by agitation. Also it is reported

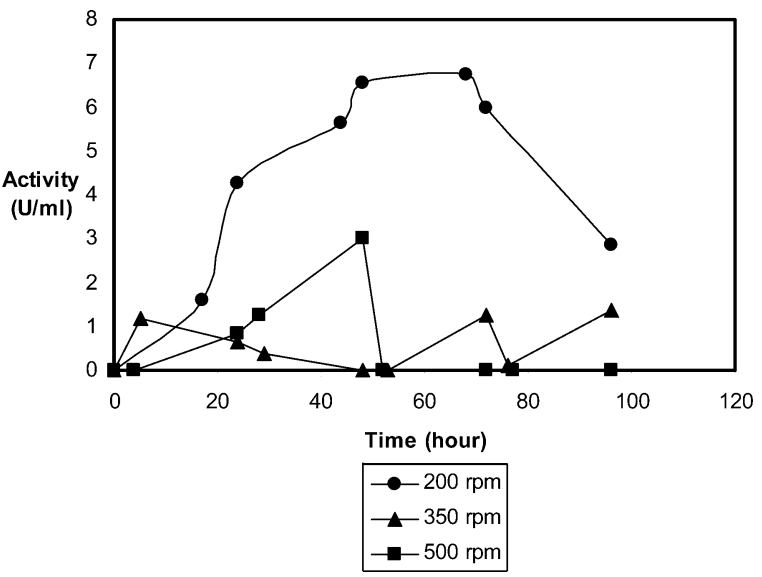

Figure 6. Polygalacturonase profiles of experiments conducted at different agitation speed of 200,350 and $500 \mathrm{rpm}$ at $30^{\circ} \mathrm{C}, 2.5 \mathrm{~L} / \mathrm{min}$ air flow rate.

in the literature by many researchers (supporting our findings) that in stirred tanks agitated up to $300 \mathrm{rpm}$ the hyrodynamic shear regimens are comparable and permit stable existence of pellets up to $2.3 \mathrm{~mm}$ in diameter (36).

Overall one can draw the final conclusions that low agitation speed $(200 \mathrm{rpm})$ was not sufficient to fullfill the high demand of oxygen, which in turn affects the carbohydrate metabolism of the culture, resulting in low biomass and small pellets. On the other hand, a medium level of agitation (350 rpm) induced bigger pellet formations with compact centers facing both oxygen and nutrient mass transfer resistance, resulting in low biomass. However, high agitation speed $(500 \mathrm{rpm})$ was also not adequate in supplying the necessary oxygen for the fast carbon metabolism due to faster growth. Chipping off (erosion) and breakage (outright rupture) mechanism resulted in small sized pellets with wide distribution.

As is well-known. biomass concentration and morphology affects the rheological properties of the fermentation broth. These effects on the rheology can be interpreted using the biomass concentration profile (Figure $4 c$ ) and viscosity data (Figure 7) along with corresponding consistency index $(K)$ and flow behavior index $(n)$ (Table 2). Non-Newtonian and pseudoplastic (shear thinning, $n<1$ ) broth rheology was observed in the case of $500 \mathrm{rpm}$ agitation speed. Conversely, broth rheology exhibited dilatant (shear thickening, $n>1$ ) behavior at the lower agitation rate $(200 \mathrm{rpm})$, and at the medium agitation speed (350 rpm) the broth was close to Newtonian $(n \approx 1.0)$. These findings reveal some important features of broth rheology: (1) At an agitation speed of $500 \mathrm{rpm}$, the $K$ value (Table 2) and biomass concentration (Figure $4 \mathrm{c}$ ) were the highest with an average pellet size of $1.08 \pm 0.42 \mathrm{~mm}$ (Figure 5b). At a low intensity agitation of $200 \mathrm{rpm}$, the biomass concentration was the lowest where the pellet size was the smallest $(0.71 \pm$ $0.35 \mathrm{~mm}$ ). This suggests that the $K$ value of broths is sensitive to both pellet size and biomass concentration, which is also supported by the study of Lopez et al. (22). They reported a sensitivity of the $K$ value of broths to pellet size but found little effect of biomass concentration on the rheology (2). The small pellets formed at the low agitation speed produced broths with $n$ values exceeding unity, i.e., shear thickening behavior. Broths containing comparably larger pellets obtained at high intensity of agitation had a shear thinning behavior (i.e., $n<1$ ). On the other hand, at an agitation speed of $350 \mathrm{rpm}$, formation of large pellets that are low in number did not affect the broth rheology significantly, Newtonian type of flow behavior was observed $(\mathrm{n} \approx 1.0)(3)$. The apparent viscosity calculated at the estimated 


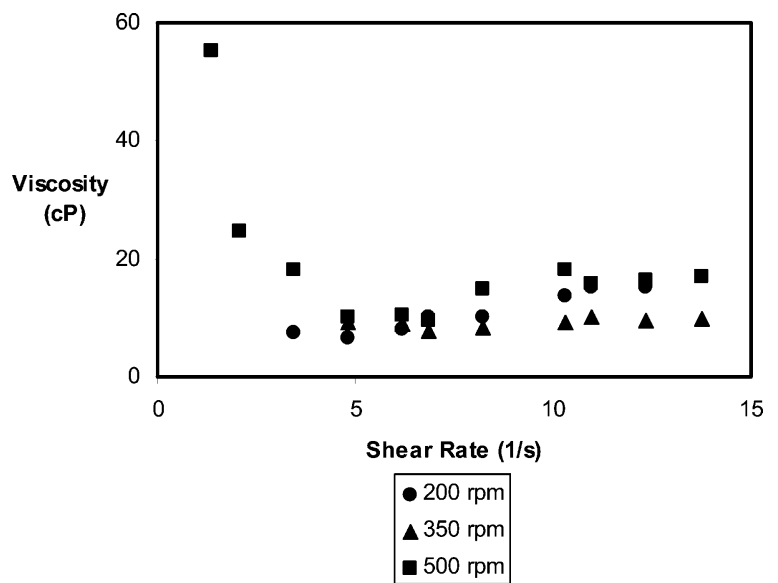

Figure 7. Changes in the viscosity of broths at different shear rates obtained at the end of fermentations carried out at 200,350, and $500 \mathrm{rpm}, 2.5 \mathrm{~L} / \mathrm{min}$ air flow rate.

Table 2. Rheological Parameters of the Broths Obtained in Fermentations Conducted at Different Agitation Speeds at $30{ }^{\circ} \mathrm{C}$, $2.5 \mathrm{~L} / \mathrm{min}$ Air Flow Rate

\begin{tabular}{|c|c|c|c|}
\hline & \multicolumn{3}{|c|}{ agitation speed (rpm) } \\
\hline & 200 & 350 & 500 \\
\hline consistency index $(K)\left(\mathrm{Pa} \cdot \mathrm{s}^{n}\right)$ & 0.005 & 0.045 & 0.1215 \\
\hline flow behavior index $(n)$ & 1.9805 & 0.9567 & 0.5033 \\
\hline \multicolumn{4}{|c|}{ constants obtained from regression analysis } \\
\hline-49.8 & & & 1.0 \\
\hline
\end{tabular}

shear rate of $13.74 \mathrm{~s}^{-1}$ (eq 4) for each agitation intensity (200, 350 , and $500 \mathrm{rpm}$ ) was found to be $15.72,9.75$, and $16.95 \mathrm{cP}$, respectively. Generally, because of the shear thickening and shear thinning behavior, the broths produced at the highest and lowest agitation rates were more viscous than the one obtained at the intermediate agitation speed where Newtonian-type flow behavior was observed. Applying regression analysis to experimental data obtained at the end of fermentation (96 h), the following relationship was found with a high correlation coefficient $\left(R^{2}=1\right)$ between $K$ value and the biomass concentration of the broths produced at different agitation speeds:

$$
Y=a X^{2}-b X+c
$$

where $Y$ is the consistency index $(K), X$ is the maximum biomass concentration (on dry basis, $\mathrm{g} / \mathrm{L}$ ), and $a, b$, and $c$ are the constants obtained from regression analysis (Table 2). The consistency index varies with biomass concentration to the power of 2. This is in good agreement with the data cited in the literature. The reported values of the consistency index $(K)$ for the broths that have power-law like behavior varies with biomass concentration, typically to the power of $0.3-3.3$ (2).

Maximum polygalacturonase activity (Figure 6) was observed at $200 \mathrm{rpm}$, demonstrating an inverse relationship with biomass formation. This was prevailing that enzyme synthesis was not growth-associated but a secondary product. Similar to $\mathrm{pH}$ effect maximum enzyme synthesis was achieved at 45-50 h. Based on this time point $(50 \mathrm{~h})$ the maximum enzyme productivities were $0.1341,0.059$, and $0.027 \mathrm{U} \mathrm{mL}^{-1} \mathrm{~h}^{-1}$ for 200,350 and $500 \mathrm{rpm}$, respectively. As with the $\mathrm{pH}$ effect, the enzyme was degraded after $70 \mathrm{~h}$ at both 200 and $350 \mathrm{rpm}$ and after $50 \mathrm{~h}$ at $500 \mathrm{rpm}$. Similarly, proteolysis could be stated as one of the reason for this degradation as discussed before.

Effect of Dissolve Oxygen Tension on Biomass, Polygalacturonase, Pellet Morphology, and Broth Rheology. In
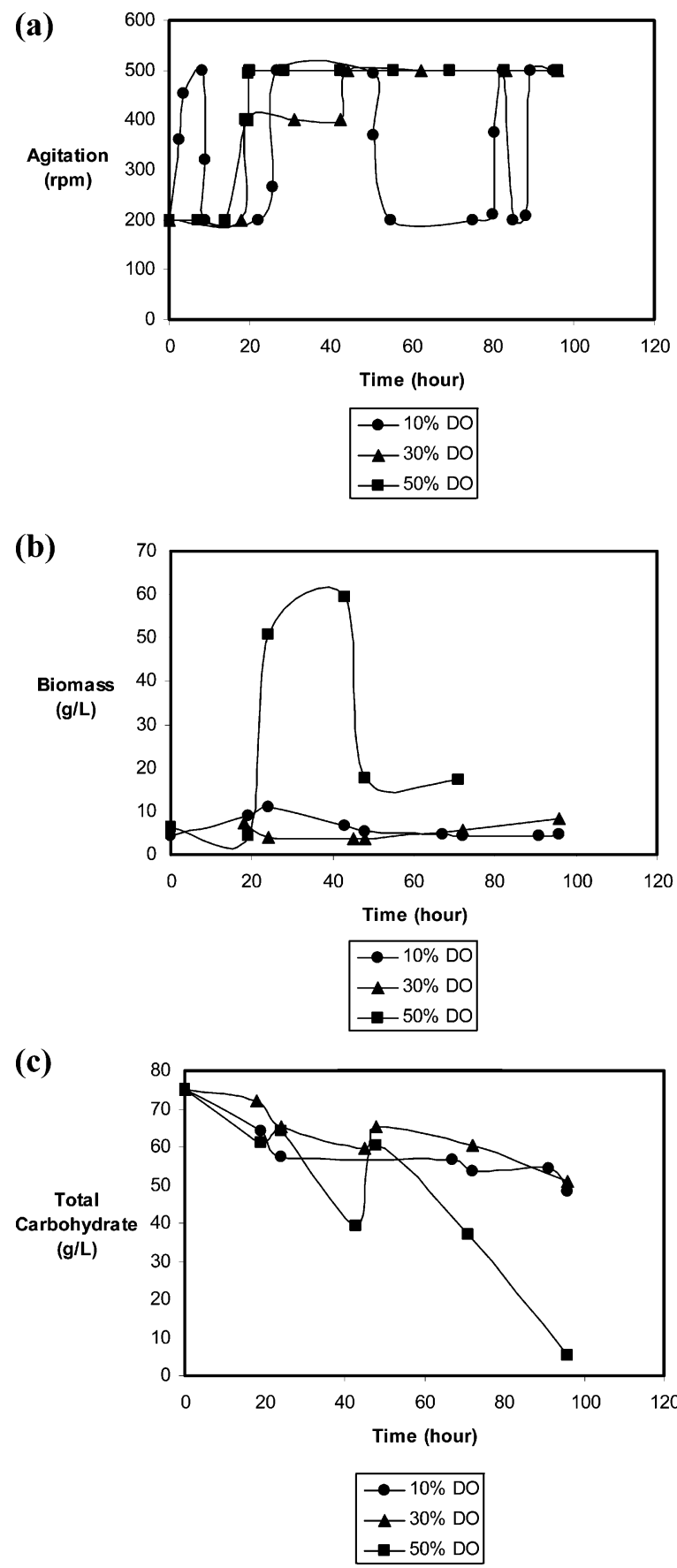

Figure 8. Profiles of (a) agitation speed, (b) biomass, and (c) carbohydrate utilization of experiments conducted at different DOTs of $10 \%, 30 \%$ and $50 \%$ at $30{ }^{\circ} \mathrm{C}, 2.5 \mathrm{~L} / \mathrm{min}$ air flow rate.

aerobic fermentations the supply of oxygen is often the ratelimiting step and satisfying oxygen demands can often constitute a large proportion of the operating and capital cost of industrial scale fermentations (2). Since oxygen plays such a detrimental role in the fungal fermentations, it was anticipated to determine the critical DOT level for the response parameters. Therefore three different saturation levels $(10 \%, 30 \%$, and 50\%) were set by the bioreactor and were controlled through cascading the agitation speed between ranges of 200-500 rpm at constant aeration rate of $2.5 \mathrm{~L} / \mathrm{min}$. All other conditions were the same as described in Materials and Methods. As can be seen from the profiles of agitation (Figure 8a), cascading first started with experiments where DOT was set to $50 \%$, followed by $30 \%$ and then $10 \%$. The system increased the agitation automatically between the prescribed ranges in order to keep the desired DOT 
levels. It was anticipated that the sooner the drop in the DOT, the sooner would cascading start and the effect of agitation would prevail on the response parameters. The highest DOT $(50 \%)$ required higher agitation levels compared to the low set DOT, which started cascating later with lower agitation speeds. All these combinations affected the response parameters at different levels.

At $50 \%$ DOT the biomass formation was 6 times higher than at $30 \%$ and $10 \%$ DOT at $45 \mathrm{~h}$ of fermentation, revealing the oxygen dependency of carbon metabolism (Figure 8b). This was also justified by the carbohydrate profiles (Figure 8c), where carbohydrates were almost completely utilized at the end of fermentation. This profile also indicated that oxygen was utilized efficiently in this particular run. However, the same could not be observed for the other two set points (30\% and 10\% DOT). For these two runs the biomass formation was very low and there was a high possibility of oxygen limitation, as can be observed from the carbohydrate profiles (not completly utilized). A significant difference in the biomass formation and carbohyrate utilization rates of these two runs could not be detected. Therefore the critical DOT level with respect to biomass formation was suggested to be in the range of $30-50 \%$ DOT. Since $40 \%$ was not checked, an exact level at this point could not be recommended.

The effect of cascading in order to keep the certain DOT levels exhibited a profound effect on the pellets sizes and distributions, as can be observed in Figure 9(a, b). At all three DOT set points a wide size distribution with different pellet numbers and sizes was observed. The average pellet sizes were $1.98 \pm 0.75,2.80 \pm 0.87$, and $1.70 \pm 0.48 \mathrm{~mm}$ for $10 \%, 30 \%$, and $50 \%$ DOT, respectively. Similary, there was significant difference in the pellet structure, with big fluffy pellets for $30 \%$, medium sized hairy pellets for $10 \%$, and small compact smooth pellets for $50 \%$ being obtained. Futhermore the number of the pellets in $1 \mathrm{~g}$ of medium varied considerably among the three set points, indicating critical information about the mechanism of pellet formation. This difference can be mainly attributed to the cascading effect through the agitation applied. The mechanism for the $50 \%$ DOT could be related to the chipping off and direct breakage of the pellets, which increased the number of pellets but reduced their sizes due to shear effect at higher agitation speed. This was in accordance with the visual observations of the pellets as described above. The mechanism for the other two was similar but with a few differences. Here for both set points agglomeration due to the lower agitation speeds were in place, increasing their sizes but reducing the numbers. This was more pronounced for set point of $30 \%$ compared to $10 \%$, where in the case of $10 \%$ DOT, oxygen limitation occurred in the medium, preventing pellet growth. This was also justified with the hairy fluffy structure that did not exhibit interior diffusion limitation otherwise (Figure 9b). However, in the case of $30 \%$ DOT in addition to the agglomeration forming larger pellets, diffusion limitation of oxygen to the interior of the pellet was observed. As a result it was conluded that the DOT set point was effective in determining the size, numbers and morphology of the pellets of A. sojae under the conditions studied.

When comparison was made with the literature, contradicting results were found relating the effect of DOT on the pellet morphology and on the production of the product of interest. For example, Gomes et al. (37) found that no difference in morphology for pellets or filaments of $A$. niger could be related to DOT levels, although the production of citric acid was enhanced by increasing DOT at different fermentation stages.
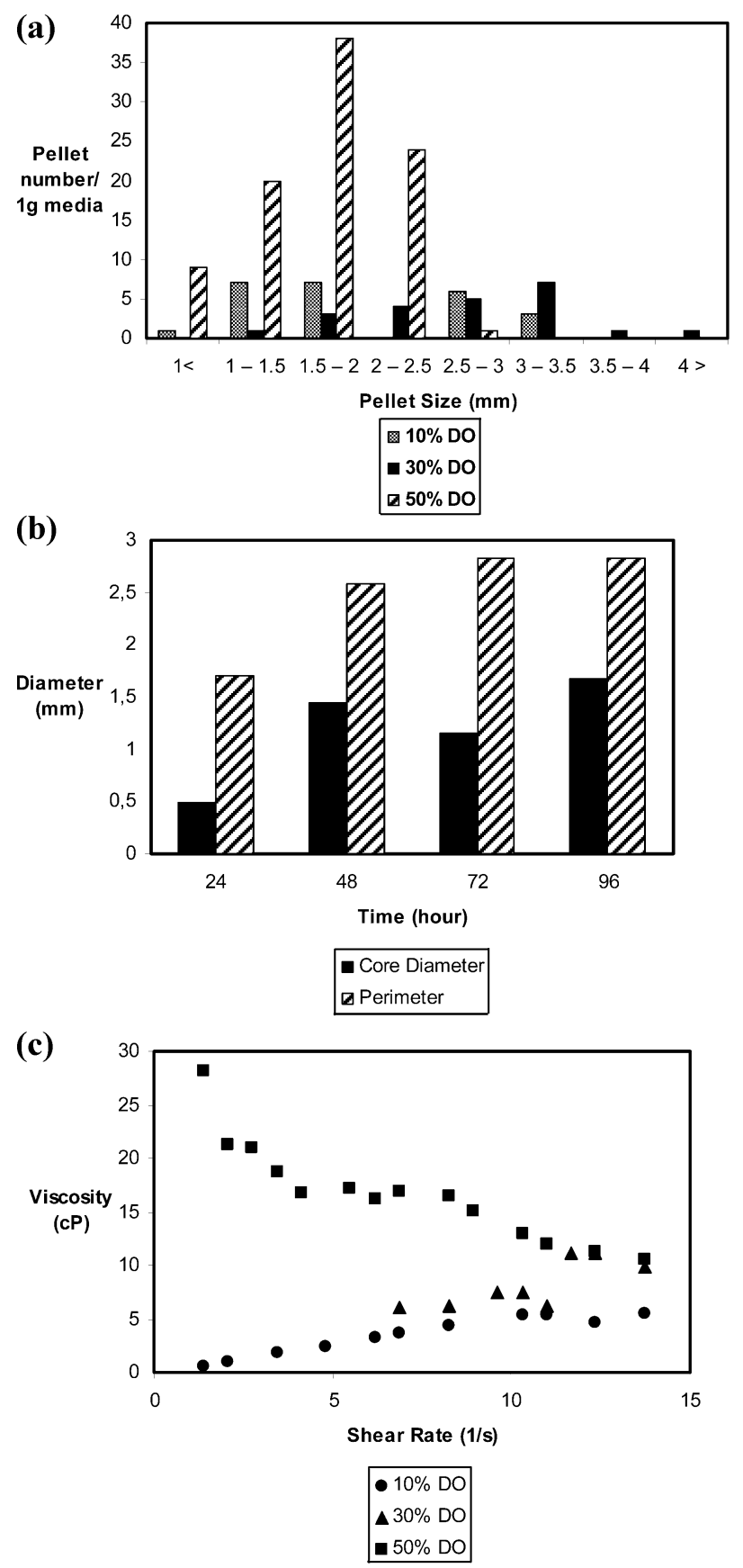

Figure 9. Profiles of (a) pellet size distribution of experiments conducted at different DOTs of $10 \%, 30 \%$, and $50 \%$ at $30{ }^{\circ} \mathrm{C}$, (b) pellet sizes during the course of fermentation run at $10 \%$ DOT, and (c) changes in the viscosity of broths at different shear rates obtained at the end of fermentations carried out at $10 \%, 30 \%$ and $50 \%$ DOT, $2.5 \mathrm{~L} / \mathrm{min}$ air flow rate.

Similarly, Carter and Bull (38) found that the morphology of A. nidulans was unaffected by DOT levels. Also no direct relationship between DOT and the morphology of Penicillum was determined by a study of van Suijdam and Metz (8). On the contrary, in a study of Higashiyama et al. (39) the fungal morphology of Mortiella alpina in the process of arachidonic acid producton appeared to be very senstive to DOT. Similarly, in the study conducted by McIntyre et al. (40), oxygen was found to be the overriding effector of morphological development of the dimorphic fungus Mucor circinelloides. With this respect the current study will be a new reference point in highlighting the effect of DOT on the morphology of a new strain (A. sojae) and contibute to the literature. 
Table 3. Rheological Parameters of the Broths Obtained in Fermentations Conducted at Different Dissolved Oxygen Tensions (DOT) at $30{ }^{\circ} \mathrm{C}, 2.5 \mathrm{~L} / \mathrm{min}$ Air Flow Rate

\begin{tabular}{llll}
\hline & \multicolumn{3}{c}{ DOT } \\
\cline { 2 - 4 } & $10 \%$ & $30 \%$ & $50 \%$ \\
\hline consistency index $(K)\left(\mathrm{Pa} \cdot \mathrm{s}^{n}\right)$ & 0.002 & 0.0035 & 0.117 \\
flow behavior index $(n)$ & 1.9712 & 1.9552 & 0.6317
\end{tabular}

Considering the rheology that is a function of the morphology, different rheological behavior was observed (Figure 9c, Table $3)$. Non-Newtonian shear thickening behavior $(n>1.0)$ was depicted in the cases of fermentation where the DOT level was kept at $10 \%$ and $30 \%$. On the other hand, when the DOT level was increased to $50 \%$, non-Newtonian shear thinning behavior $(n<1.0)$ was dominant. Using higher DOT level produced smaller and compact spherical pellets, whereas large and fluffy pellets were obtained at $10 \%$ and $30 \%$ DOT. Despite some debates in the literature about the effect of DOT on the morphology and rheology of the fermentation broth, it appeared that A. sojae morphology was sensitive to DOT in the process of polygalacturonase production and that the rheology of the broth was directly affected by the morphology.

The effect of different DOT set points on polygalacturonase activity is presented in Figure 10. In contrast to biomass formation highest enzyme activity was observed at 10\% DOT, confirming the inverse relation ship between biomass and enzyme production. Obviously, at this DOT level (10\%) the resources were directed toward enzyme synthesis rather than biomass formation. This could be partly related to the enzyme synthesis metabolism, that oxygen was not a limiting step for this process and also to the morphology of the culture where medium sized pellets with hairy region could make better use of the necessary oxygen and nutrient available in the medium. The maximum enzyme activity achieved at $65 \mathrm{~h}$ at $10 \%$ DOT was 4 and 6 times higher than at 30\% and 50\% DOT, repectively. Again a degradation of the enzyme after this time point for all three set points was due to possible proteolysis as discussed before. Therefore, on the basis of these findings if the primary goal is to produce the polygalacturonase enzyme, then it is recommended to set the DOT to $10 \%$, which not only will maximize the product yield but also reduce the power input due to delayed cascading process, eventually being reflected in the low operational cost. The final $\mathrm{pH}$ of all three runs was around $\mathrm{pH} 4$.

A direct relationship between the DOT levels and the production of citric acid by $A$. niger, penicillin production by Pe. chrysogenum, and arachidonic acid production by Mortiella alpina has been established in a number of studies $(37,39)$. Also in the current study a critical DOT level $(50 \%)$ for biomass formation different from the critical level (10\%) for maximum polygalacturonase production was determined .

\section{Conclusions}

Overall the current study demonstrated that $\mathrm{pH}$, agitation speed, and DOT levels are critical key parameters influencing the morphology, broth rheology, and polygalacturonase production of A. sojae in a bioreactor. This study did not only provide novel information about the production conditions of polygalacturonase by a new strain, $A$. sojae, but also provided new numerical data on morphological (pellet size and number) and rheological $(K, n)$ parameters that can be used in the mathematical modeling and scaling up processes of common fungal fermentations. These data can be used further in the overall process design and the subsequent down stream process.

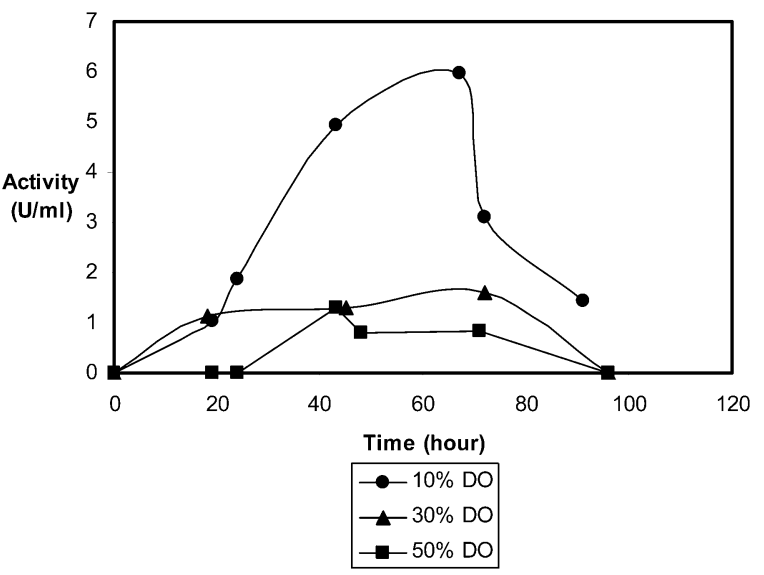

Figure 10. Polygalacturonase profiles of experiments conducted at different dissolved oxygen tensions (DOT) of $10 \%, 30 \%$ and $50 \%$ at $30{ }^{\circ} \mathrm{C}, 2.5 \mathrm{~L} / \mathrm{min}$ air flow rate.

\section{Acknowledgment}

Financial support of Izmir Institute of Technology through the projects IYTE 2004,04 and IYTE 2004,08 is gratefully acknowledged.

\section{References and Notes}

(1) Mitard, A.; Riba, J. P. Morphology and growth of Aspergillus niger ATCC 26036 cultivated at several shear rates. Biotechnol. Bioeng. 1987, 32, 835-840.

(2) Papagianni, M. Fungal morphology and metabolite production in submerged mycelial processes. Biotechnol. Adv. 2004, 22, 189259.

(3) Pedersen, A. G.; Bundgaard-Nielsen, M.; Nielsen, J.; Villadsen, J.; Hassager, O. Rheological characterization of media containing Penicillium chrysogenum. Biotechnol. Bioeng. 1993, 41, 162.

(4) Reichl, U.; King, R.; Gilles, E. D. Characterization of pellet morphology during submerged growth of Streptomyces tendae by image analysis. Biotechnol. Bioeng. 1991, 39, 164-170.

(5) Li, Z. J.; Shukla, V.; Fordyce, A. P.; Pedersen, A. G.; Wenger, K. S.; Marten, M. R. Fungal morphology and fragmentation behavior in a fed-batch Aspergillus oryzae fermentation at the production scale. Biotechnol. Bioeng. 2000, 70, 300-312.

(6) Pazauki, M.; Panda, T. Understanding the morphology of fungi. Bioprocess Eng. 2000, 22, 127-143.

(7) Metz, B.; Kossen, N. W. F. The growth of molds in the form of pellets, A literature review. Biotechnol. Bioeng. 1977, 19, 781-799.

(8) Suijdam, J. C.; Metz, B. Influence of engineering variables upon the morphology of filamentous molds. Biotechnol. Bioeng. 1981, 23, 111-148.

(9) Atkingson, B.; Daoud, L. S. Microbial flogs and flocculation in fermentation process engineering. Adv. Biochem. Eng. 1976, 4, 41124.

(10) Zhaou, Y.; Du, J.; Tsao, G. T. Mycelial pellet formation by Rhizopus oryzae ATCC 20344. Appl. Biochem. Biotechnol. 2000, 84-86, 779-789.

(11) Ustok, F. I.; Tari, C.; Gogus, N. Solid-state production of polygalacturonase by Aspergillus sojae ATCC 20235. J. Biotechnol. 2007, 127, 322-334

(12) Gögus, N.; Tari, C.; Oncü, S.; Unluturk, S.; Tokatli, F. Relationship between morphology, rheology and polygalacturonase production by Aspergillus sojae ATCC 20235 in submerged cultures. Biochem. Eng. J. 2006, 32, 171-178.

(13) Tari, C.; Gögus, N.; Tokatli, F. Optimization of biomass, pellet size and polygalacturonase production by Aspergillus sojae ATCC 20235 using response surface methodology. Enzyme Microb. Technol. 2007, 40, 1108-1116.

(14) Patil, S. R.; Dayanand, A. Optimization of process for the production of fungal pectinases from deseeded sunflower head in submerged and solid-state conditions. Bioresource Technol. 2006, 97, 2340-2344.

(15) Kaur, G.; Kumar, S. Satyanarayana. Production, characterization and application of a thermostable polygalacturonase of a thermophilic 
mould Sporotrichum thermophile Apinis. Bioresource Technol. 2004, 94, 239-243.

(16) Jayani, R. S.; Saxena, S.; Gupta, R. Microbial pectinolytic enzymes: A review. Process Biochem. 2005, 40 (9), 2931-2944.

(17) Panda, T.; Naidu, G. S. N.; Sinha, J. Multiresponse analysis of microbiological parameters affecting the production of pectolytic enzymes by Aspergillus niger: a statistical view. Process Biochem. 1999, 35, 187-195.

(18) Lowry, O. H.; Rosebrough, N. J.; Farr, A. L.; Randall, J. R. Protein measurement with the folin phenol reagent. J. Biol. Chem. 1951, $193,265-275$.

(19) DuBois, M.; :Gilles, K. A.; Hamilton, J. K.; Rebers, P. A.; Smith, F. Colorimetric method for determination of sugars and related substances. Anal. Chem. 1956, 28 (3), 350-356.

(20) Park, J. P.; Kim, Y. M.; Kim, S. W.; Hwang, H. J.; Cho, Y. J.; Lee, Y. S.; Song, C. H.; Yun, J. W. Effect of aeration rate on the mycelial morphology and exo-biopolymer production in Cordyceps militaris. Process Biochem. 2002, 37, 1257-1262.

(21) Cox, P. W.; Thomas, C. R. Classification and measurement of fungal pellets by automated image analysis. Biotechnol. Bioeng. 1992, 39, 945-952.

(22) Lopez, J. L. C.; Perez, J. A. S.; Sevilla, J. M. F.; Porcel, E. M. J.; Chisti, Y. Pellet morphology, culture rheology and lovastatin production in cultures of Aspergillus terreus. J. Biotechnol. 2005, $116,61-77$.

(23) More Solution to Sticky Problems: A Guide to Getting More from Your Brookfield Viscometer; Brookfield Engineering Laboratories, Inc. : Middleboro, MA, 2000.

(24) Metzner, A. B. Rheology of suspensions in polymeric liquid. Soc. Rheol. 1985, 29 (6), 739-775.

(25) Rao, M. A. Rheology of Fluid and Semisolid Foods: Principles and Applications; Aspen Publishers: Gaithersburg, MD, 1999.

(26) Cho, Y. J.; Hwang, H. J.; Kim, S. W.; Song, C. H.; Yun, J. W. Effect of carbon source and aeration rate on broth rheology and fungal morphology during red pigment production by Paecilomyces sinclairii in batch bioreactor. J. Biotechnol. 2002, 95, 13-23.

(27) Benett, J. W. Aspergillus and Koji: History, practice and molecular biology. Soc. Ind. Enzymes News 2001, 51, 65-71.

(28) Fang, Q. H.; Zhong, J. J. Effect of initial $\mathrm{pH}$ on production of ganoderic acid and polysaccharide by submerged fermentation of Ganoderma lucidum. Process Biochem. 2002, 37, 769-774.

(29) Papagianni, M.; Mattey, M.; Kristiansen, B. Morphology and citric acid production of Aspergillus niger PM1. Biotechnol. Lett. 1994, 16, 929-934.
(30) Mattey, M. The production of organic acids. CRC Crit. Rev. Biotechnol. 1992, 12, 87-132.

(31) Carlsen, M.; Spöhr, A. B.; Nielsen, J.; Villadsen, J. Morphology and physiology of an $\alpha$-amylase producing strain of Aspergillus oryzae during batch cultivations. Biotechnol. Bioeng. 1995, 49, 266276.

(32) Mantzouridou, F.; Roukas, T.; Kotzekidou, P. Effect of the aeration rate and agitation speed on $\beta$-carotene production and morphology of Blakeslea trispora in a stirred tank reactor: mathematical modeling. Biochem. Eng. J. 2002, 10, 123-135.

(33) Papagianni, M.; Mattey, M.; Kristiansen, B. Citric acid production and morphology of Aspergillus niger as functions of the mixing intensity in a stirred tank and a tubular loop bioreactor. Biochem. Eng. J. 1998, 2, 197-205.

(34) Jüsten, P.; Paul, G. C.; Nienow, A. W.; Thomas, C. R. Dependence of Penicillium chrysogenum growth, morphology, vacuolation, and productivity in fed-batch fermentations on impeller type and agitation intensity. Biotechnol. Bioeng. 1998, 59, 762-775.

(35) Nielsen, J.; Johansen, C. L.; Jacobsen, M.; Krabben, P.; Villadsen, J. Pellet formation and fragmentation in submerged cultures of Penicillum chrysogenum and its relation to penicilin production. Biotechnol. Prog. 1995, 11, 93-98.

(36) Porcel, E. M. R.; Lopez, J. L. C.; Perez, J. A. S.; Sevilla, J. M. F.; Chisti, Y. Effects of pellet morphology on broth rheology in fermentations of Aspergillus terreus. Biochem. Eng. J. 2005, 26, $139-144$.

(37) Gomez, R.; Schnabel, I.; Garrido, J. Pellet growth and citric acid yield of Aspergillus niger 110. Enzyme Microb. Technol. 1988, 10, $188-191$.

(38) Carter, B. L. A.; Bull, A. T. The effect of oxygen tension in the medium on the morphology and growth kinetics of Aspergillus nidulans. J. Gen. Microbiol. 1971, 65, 265-273.

(39) Higashiyama, K.; Murakami, K.; Tsujimura, H.; Matsumoto, N.; Fujikawa, S. Effects of dissolved oxygen on the morphology of an arachidonic acid production by Mortiella alpina 1S-4. Biotechnol. Bioeng. 1999, 63, 442-448.

(40) McIntyre, M.; Breum, J.; Arnau, J.; Nielsen, J. Growth physiology and dimorphism of Mucor circinelloides (syn. racemosus) during submerged batch cultivation. Appl. Microbiol. Biotechnol. 2002, 58 , 495-502.

Received March 23, 2007. Accepted May 21, 2007.

BP070079C 